\title{
Mediastinite após cirurgias valvares cardíacas: revisão integrativa
}

\author{
Ana Sara Mendes Teixeira ${ }^{1}$, \\ Laura Menezes Silveira², \\ Angelita Maria Stabile ${ }^{3}$,
} Carina Aparecida Marosti Dessotte ${ }^{4}$

\section{RESUMO}

A mediastinite é uma infecção do tecido conjuntivo do mediastino, pouco estudada em pacientes submetidos às cirurgias de correção de valvopatias cardíacas. $\mathrm{O}$ objetivo deste estudo foi identificar na literatura as evidências relacionadas à mediastinite em pacientes submetidos às cirurgias para correção de valvopatias cardíacas. Foi realizado uma revisão integrativa com buscas, em cinco bases de dados eletrônicas: LILACS, PubMed, Scopus, EBSCOhost e Web of Science. De acordo com os critérios estabelecidos, quatro artigos foram incluídos para a análise e mostraram aspectos sobre a mediastinite, como: preditores de mortalidade no pré-operatório, contaminação de materiais no intraoperatório e técnica de abordagem cirúrgica. Estudos com enfoque específico à ocorrência de mediastinite nestes pacientes, podem instrumentalizar a equipe de saúde e melhorar a implementação das ações na prática clínica, prevenindo complicações.

Descritores: Mediastinite; Valvas Cardíacas; Fatores de Risco; Infecção da Ferida Cirúrgica; Enfermagem Cirúrgica.

\footnotetext{
${ }^{1}$ Enfermeira. Ribeirão Preto, SP, Brasil. E-mail: ana.sara.teixeira@usp.br.

2 Enfermeira, Mestre em Enfermagem Fundamental. Discente do Programa de Pós-Graduação em Enfermagem Fundamental, nível Doutorado, da Escola de Enfermagem de Ribeirão Preto da Universidade de São Paulo. Ribeirão Preto, SP, Brasil. E-mail: lauramsilveira@usp.br.

${ }^{3}$ Enfermeira, Doutora em Fisiologia. Professora Doutora da Escola de Enfermagem de Ribeirão Preto da Universidade de São Paulo. Ribeirão Preto, SP, Brasil. E-mail: angelita@eerp.usp.br.

${ }^{4}$ Enfermeira, Doutora em Enfermagem. Professora Doutora da Escola de Enfermagem de Ribeirão Preto da Universidade de São Paulo. Ribeirão Preto, SP, Brasil. E-mail: camarosti@eerp.usp.br.
}

\section{Como citar esse artigo:}

Teixeira ASM, Silveira LM, Stabile AM, Dessotte CAM. Mediastinite após cirurgias valvares cardíacas: revisão integrativa. Rev. Eletr. Enf. [Internet]. 2018 [acesso em: ];20:v20a23. Disponível em:

https://doi.org/10.5216/ree.v20.47822. 


\section{INTRODUÇÃO}

As Doenças Cardiovasculares (DCV) constituem a principal causa de morbimortalidade, tanto nos países em desenvolvimento como nos desenvolvidos. Em 2014, no Brasil, a taxa de mortalidade por doenças do aparelho circulatório em todas as faixas etárias foi de $27,73 \%$, principalmente, a partir dos 50 anos. O número de óbitos por doenças do aparelho circulatório foi de 340.284 no Brasil e no Estado de São Paulo de 82.592 óbitos, o que representa o maior índice de mortalidade do país ${ }^{(1)}$.

Embora seja possível observar um avanço tecnológico nos tratamentos minimamente invasivos, a cirurgia cardíaca ainda é o tratamento de escolha para muitos pacientes com DCV.

A cirurgia cardíaca com circulação extracorpórea (CEC) provoca grande impacto na homeostasia do organismo. Neste sentido, manejar o paciente no pós-operatório (PO) inclui identificar as alterações que representam complicações e àquelas que fazem parte de uma recuperação fisiológica. Assim, as principais complicações no PO de cirurgia cardíaca vêm sendo abordadas devido à complexidade envolvida. Estas complicações podem estar relacionadas ao tempo de internação pré-operatória; às comorbidades pré-existentes; aos hábitos de vida inadequados (tabagismo, sedentarismo); idade avançada; à condição nutricional prévia (desnutrição ou obesidade); tipo de medicamento utilizado no pré-operatório e fatores de risco inerentes ao procedimento anestésico-cirúrgico(2-5).

Dentre as complicações PO, destacamos neste estudo a mediastinite. A prevalência de mediastinite no PO varia de 0,4 a 5\%, em média está presente de um a $2 \%$ em pacientes cardiopatas e, apesar da baixa prevalência, tem elevadas taxas de mortalidade, que podem variar entre 14 a $47 \%{ }^{(6)}$. Além disso, o tempo de internação pode ser maior, ocasionando o aumento dos custos aos serviços de saúde e a piora da qualidade de vida do paciente ${ }^{(5)}$.

A mediastinite é uma infecção e/ou inflamação do tecido conjuntivo do mediastino. De acordo com o Centro de Controle e Prevenção de Doenças (CDC), os critérios diagnósticos para mediastinite após esternotomia são instabilidade esternal e cultura bacteriana positiva do mediastino ${ }^{(7)}$. Pode ocorrer até os 30 primeiros dias do PO, manifestada pela dor ou instabilidade do esterno, associados a drenagem purulenta através da área retroesternal, hemocultura positiva ou da secreção drenada e aumento do mediastino em exame de imagem; evidência de infecção retroesternal durante a operação ou análise histológica ${ }^{(8)}$.

Diversos estudos têm investigado os fatores de risco para mediastinite em pacientes submetidos à cirurgia de Revascularização do Miocárdio (CRVM), ressalta-se ainda que, um escore de risco para mediastinite foi elaborado para esses pacientes ${ }^{(7)}$. Contudo, poucos estudos investigaram a ocorrência da mediastinite em pacientes submetidos às cirurgias cardíacas para correção de valvopatias exclusivamente, ocasionando falta de evidências. Acreditamos que as precauções-padrão utilizadas para a prevenção de infecção, especificamente a mediastinite, não seriam suficientes para esses pacientes, considerando que a cirurgia cardíaca é de grande porte, e os pacientes são submetidos à CEC.

Com o aumento da expectativa de vida, a prevalência das valvopatias graves se eleva, podendo acometer até $2 \%$ dos indivíduos acima dos 65 anos $^{(9)}$, e muitas vezes o procedimento cirúrgico é indicado. No entanto, a sobrevida após o aparecimento dos sintomas é baixa, sendo de $60 \%$, em um ano, e $32 \%$, em cinco anos ${ }^{(10)}$.

Conhecer as evidências disponíveis sobre a ocorrência da mediastinite nos pacientes submetidos a cirurgias valvares, poderá auxiliar no planejamento dos cuidados destinados a estes pacientes no perioperatório e, 
principalmente na prevenção desta complicação. Assim, este estudo teve como objetivo identificar na literatura as evidências associadas à ocorrência de mediastinite em pacientes submetidos a cirurgias para correção de valvopatias.

\section{MÉTODO}

Revisão integrativa, realizada em seis etapas: identificação do tema e da questão de pesquisa; estabelecimento de critérios para inclusão e exclusão de estudos e da busca na literatura; definição das informações a serem extraídas dos estudos selecionados e categorização dos estudos; avaliação dos estudos incluídos; interpretação dos resultados e apresentação da síntese do conhecimento ${ }^{(11)}$.

A questão norteadora foi elaborada com auxílio da estratégia "PICO", que representa um acrônimo para Paciente, Intervenção, Comparação e "Outcomes" (desfecho), com vistas à localização de melhores evidências. Neste estudo, o acrônimo foi utilizado considerando "P" - Pacientes com valvopatias, "I" - Cirurgias para correção de valvopatias, " $\mathrm{C}$ "- Não aplicável, e "O"- Mediastinite. A questão norteadora centrou-se em: "Quais as evidências sobre a ocorrência de mediastinite em pacientes submetidos às cirurgias para correção de valvopatias?".

As buscas foram realizadas em cinco bases de dados eletrônicas: Literatura Latino-Americana e do Caribe em Ciências da Saúde (LILACS), US National Library of Medicine, National Institutes of Health (PubMed), Scopus, EBSCOhost e Web of Science. Foram utilizados descritores controlados extraídos do Descritores em Ciências da Saúde (DeCS) e do Medical Subject Headings (MeSH): Mediastinitis (Mediastinite), Aortic Valve (valva aórtica), Pulmonary Valve (Valva Pulmonar), Tricuspid Valve (Valva Tricúspide), Mitral Valve (Valva mitral), Thoracic Surgery (Cirurgia Torácica), Surgery (Cirurgia), Risk factors (fatores de risco) além de palavras-chave, Valve Replacement (troca de valva), Surgical infection (infecção cirúrgica) e Cardiac Valve (valva cardíaca). Em todos os cruzamentos foram utilizados os operadores booleanos "AND", “OR", e "AND NOT".

Para a inclusão dos artigos foram considerados os critérios: artigos originais que atenderam a questão norteadora, desenvolvidos com indivíduos acima de 18 anos, publicados em periódicos na língua inglesa, espanhola, francesa e portuguesa, publicados em qualquer período.

Para exclusão dos artigos, adotou-se os critérios: publicações que não se enquadravam na classificação do nível de evidências utilizadas ${ }^{(12)}$, editoriais, carta ao editor, dissertações, casos clínicos, teses, revisões de literatura e artigos que possuíam texto indisponível.

A busca foi realizada em dezembro de 2016. Para a coleta de dados dos estudos elegíveis, adaptou-se um instrumento validado, que engloba a identificação do artigo, os autores, o tipo de publicação, o detalhamento metodológico, o detalhamento amostral, a intervenção estudada, os resultados e as recomendações/conclusões ${ }^{(13)}$. As publicações selecionadas foram classificadas de acordo com o delineamento metodológico e o nível de evidência. A síntese dos dados será apresentada de forma descritiva.

\section{RESULTADOS}

As buscas realizadas nas bases de dados identificaram 530 artigos sobre a temática, sendo que 180 artigos estavam duplicados e foram excluídos. De acordo com os critérios de elegibilidade estabelecidos, 350 estudos 
foram rastreados pela leitura do título e resumo e selecionou-se 32 artigos para análise do texto na íntegra. Desses, 28 foram desconsiderados pelos motivos: 25 não atendiam a questão norteadora, pois generalizaram os resultados com base em amostras de pacientes submetidos a cirurgias cardíacas diversas, tais como CRMV e correção de doenças da aorta; um estudo secundário, e dois apresentavam textos indisponíveis. Vale ressaltar que os autores correspondentes dos artigos indisponíveis foram contatados por e-mail, porém não se obteve resposta. A trajetória para a seleção das publicações pode ser visualizada na Figura 1.

Figura 1: Processo de seleção de artigos na revisão integrativa. Ribeirão Preto, SP, Brasil, 2017.

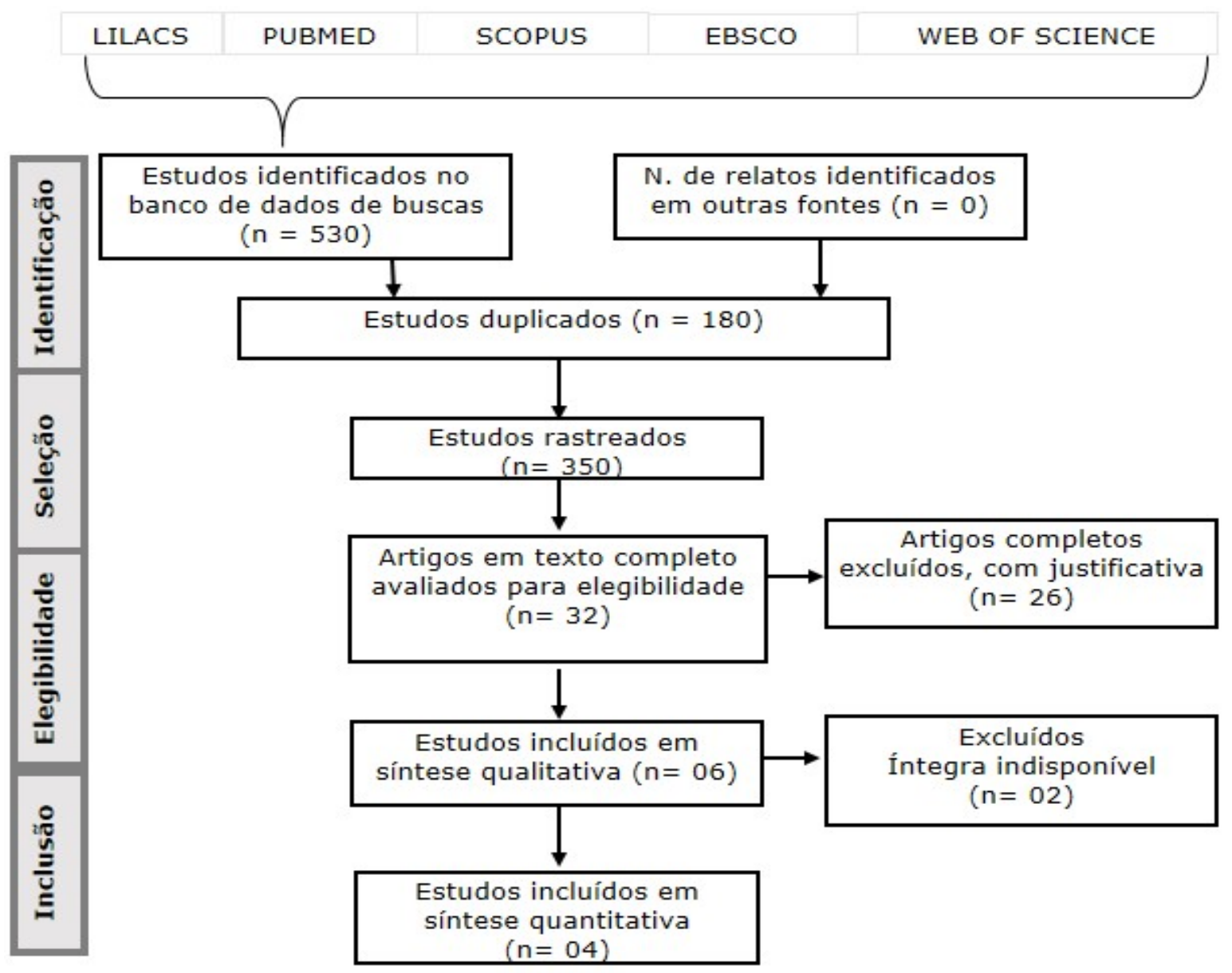

Fonte: Elaborado pelos autores segundo as recomendações de Moher et al., (2015)(14).

A amostra final foi composta por quatro artigos publicados na língua inglesa, sendo um realizado nos Estados Unidos, um na Suécia, um no Japão e um na Itália.

O Quadro 1 apresenta as características dos artigos selecionados para esta revisão, segundo autor, o ano de publicação, o periódico, a base de dados, o escopo de publicação, o nível de evidência e o país de origem. 


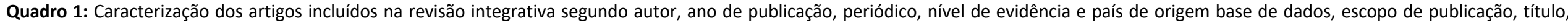
periódico, base de dados, população/amostra, delineamento e resultados. Ribeirão Preto, SP, Brasil, 2017.

\begin{tabular}{|c|c|c|c|c|c|}
\hline & $\begin{array}{c}\text { Autor, ano publicação, } \\
\text { nível de evidência, país } \\
\text { de origem, escopo de } \\
\text { publicação }\end{array}$ & $\begin{array}{c}\text { Título do manuscrito, } \\
\text { periódico e base de dados }\end{array}$ & Delineamento & População/ Amostra & Resultados associados à Mediastinite \\
\hline 1 & $\begin{array}{l}\text { Thourani et al., } \\
\text { 2011(15), IV, Estados } \\
\text { Unidos, Medicina. }\end{array}$ & $\begin{array}{l}\text { Outcomes of Surgical Aortic } \\
\text { Valve Replacement in High-Risk } \\
\text { Patients: A Multiinstitutional } \\
\text { Study, Ann Thorac Surg, } \\
\text { Embase }\end{array}$ & $\begin{array}{l}\text { Estudo de coorte retrospectiva, no período de } \\
\text { seis anos (2002-2007), em quatro instituições. } \\
\text { O estudo buscou avaliar a morbidade e } \\
\text { mortalidade a curto e médio prazo de } \\
\text { pacientes submetidos a correção valvar } \\
\text { aórtica, segundo o escore da "Society of } \\
\text { Thoracic Surgeons predicted risk of mortality". }\end{array}$ & $\begin{array}{c}\text { Constituída por } 159 \text { pacientes } \\
\text { submetidos a troca primária } \\
\text { de válvula aórtica. }\end{array}$ & $\begin{array}{l}\text { Os autores não encontraram preditores pré- } \\
\text { operatórios estatisticamente significativos para } \\
\text { mortalidade hospitalar, mas identificaram que } \\
\text { um paciente }(0,6 \%) \text { evoluiu com mediastinite } \\
\text { no PO. }\end{array}$ \\
\hline 2 & $\begin{array}{l}\text { Larsson et al., 2015(16), } \\
\text { IV, Suécia, Medicina }\end{array}$ & $\begin{array}{l}\text { Bacterial contamination of } \\
\text { suction catheter tips during } \\
\text { aortic valve replacement } \\
\text { surgery: a prospective } \\
\text { observational cohort study, } \\
\text { Patient Safety in Surgery, } \\
\text { Embase }\end{array}$ & $\begin{array}{l}\text { Estudo descritivo, onde o objetivo foi } \\
\text { investigar a presença de contaminação do } \\
\text { cateter de sucção utilizado no intraoperatório } \\
\text { de cirurgias de substituição da valva aórtica. } \\
\text { Foram coletados dois cateteres de sucção para } \\
\text { cada cirurgia. Foram utilizados cinco cateteres } \\
\text { de controle. }\end{array}$ & $\begin{array}{l}\text { A amostra foi constituída por } \\
\text { cateteres de sucção utilizados } \\
\text { em } 25 \text { cirurgias de } \\
\text { substituição valvar em } \\
\text { adultos sem infecção em } \\
\text { curso, durante o mês de } \\
\text { março de } 2014 \text {. }\end{array}$ & $\begin{array}{l}\text { A contaminação bacteriana em } 20 \text { de } 25 \text { casos } \\
\text { (80\%), sendo em cinco ( } 20 \%) \text {, tanto o primeiro } \\
\text { quanto o segundo cateter estavam } \\
\text { contaminados. A bactéria mais frequente } \\
\text { encontrada nos cateteres de sucção foi a } \\
\text { estafilococos coagulase-negativa e foi } \\
\text { associada à mediastinite. Os cateteres de } \\
\text { controle não apresentaram contaminação. }\end{array}$ \\
\hline 3 & $\begin{array}{l}\text { Nishi et al., 2015(17), IV, } \\
\text { Japão, Medicina. }\end{array}$ & $\begin{array}{l}\text { Propensity-matched analysis of } \\
\text { minimally invasive mitral valve, } \\
\text { Surgical Today, Pubmed } \\
\text { repair using a nationwide } \\
\text { surgical database }\end{array}$ & $\begin{array}{l}\text { Estudo de Coorte, retrospectivo. O período } \\
\text { analisado foi de } 2008 \text { a 2012. O objetivo era } \\
\text { realizar comparações sobre a eficácia, } \\
\text { segurança de duas técnicas cirúrgicas para } \\
\text { correção de valvopatia mitral - mini- } \\
\text { toracotomia a direita (MTD) e a esternotomia } \\
\text { mediana (EM). As características } \\
\text { sociodemográficas, clínicas e o desfecho dos } \\
\text { pacientes também foram analisados. }\end{array}$ & $\begin{array}{c}\text { Constituída por } 6137 \\
\text { pacientes provenientes de } \\
210 \text { instituições japonesas. Os } \\
\text { pacientes foram alocados em } \\
2 \text { grupos: } 756 \text { pacientes } \\
\text { submetidos a MTD e } 5381 \\
\text { pacientes submetidos a EM. }\end{array}$ & $\begin{array}{l}\text { As taxas de mediastinite e tempo de } \\
\text { permanência na UTI e de PO total até a alta } \\
\text { hospitalar foram inferiores no grupo de } \\
\text { pacientes submetidos à MTD. }\end{array}$ \\
\hline 4 & $\begin{array}{c}\text { Sansone et al., } 2012^{(18)} \\
\text { VI, Itália, Medicina. }\end{array}$ & $\begin{array}{l}\text { Right minithoracotomy versus } \\
\text { full sternotomy for the aortic } \\
\text { valve replacement: Preliminary } \\
\text { results, Heart Lung and } \\
\text { Circulation, Pubmed }\end{array}$ & $\begin{array}{l}\text { Estudo de coorte retrospectiva, cujo objetivo } \\
\text { foi de comparar a evolução pós-operatória de } \\
\text { um grupo de pacientes que foi submetido à } \\
\text { MTD com outro grupo submetido à EM para } \\
\text { correção cirúrgica de troca de válvula aórtica. }\end{array}$ & $\begin{array}{l}\text { Foram selecionados dois } \\
\text { grupos de pacientes sendo: } \\
50 \text { pacientes sob via MTD e } \\
50 \text { pacientes via EM. }\end{array}$ & $\begin{array}{l}\text { Nenhum paciente submetido à MTD } \\
\text { apresentou mediastinite e todos tiveram alta } \\
\text { hospitalar. No grupo de pacientes submetidos } \\
\text { à EM, } 2 \% \text { evoluíram com mediastinite e a } \\
\text { mortalidade nesse grupo foi de } 4 \% \text {. }\end{array}$ \\
\hline
\end{tabular}




\section{DISCUSSÃO}

A síntese qualitativa dos quatro estudos que atenderam à pergunta norteadora, mostrou que as evidências sobre mediastinite em pacientes submetidos à cirurgia de correção de valvopatias podem estar associadas a aspectos como a contaminação de materiais no intraoperatório e a técnica de abordagem cirúrgica. Adicionalmente, em um dos estudos, os preditores de mortalidade encontrados no pré-operatório não foram associados a mortalidade e à ocorrência de mediastinite.

Destaca-se que durante a fase de seleção e análise dos estudos foram encontrados diversos delineamentos que incluíram simultaneamente em suas amostras pacientes submetidos a CRVM e cirurgias valvares, e que apresentaram complicações como a mediastinite ${ }^{(19-21)}$. Estes estudos destacaram como fatores para a ocorrência de mediastinite a idade avançada, o diabetes mellitus, a obesidade, a cirurgia de revascularização prévia ${ }^{(22)}$, a fração de ejeção diminuída, o uso de balão intraórtico, a necessidade de hemodiálise, níveis de creatinina sérica acima de $2,26 \mathrm{mg} / \mathrm{dl}$ e intervenção vascular extracardíaca ${ }^{(23)}$.

As causas da mediastinite podem ser multifatoriais, tornando-a uma complicação infecciosa grave, que envolve o espaço mediastinal e o esterno. A manipulação excessiva do paciente internado e o uso prolongado de cateteres para acesso venoso central, hemodiálise, punções venosas e arteriais, pacientes com imunodeficiências ou em mau estado de nutrição, também podem favorecer a infecção(23).

Desse modo, evidencia-se a carência de estudos específicos voltados para a ocorrência de mediastinite em pacientes submetidos à cirurgias valvares, e a necessidade de exploração deste conteúdo, para ampliação do conhecimento e reavaliação da prática clínica.

Um estudo ${ }^{(15)}$ foi desenvolvido a partir da construção de um modelo preditivo de mortalidade em pacientes submetidos a cirurgias valvares, e mostrou que os preditores pré-operatórios (doença vascular periférica, acidente vascular cerebral, insuficiência renal, insuficiência cardíaca classe III e IV, cirurgia prévia de revascularização do miocárdio) não foram estatisticamente significativos para a mortalidade hospitalar. Entretanto, o estudo mostrou que um paciente evoluiu com mediastinite como complicação pós-operatória, muitas vezes associado ao risco de morte ${ }^{(6)}$.

Em contrapartida, outros estudos sobre a mediastinite após cirurgias cardíacas indicam que idade acima de 75 anos, obesidade (IMC>35), acidente vascular cerebral prévio, diabetes mellitus e parada cardíaca durante o procedimento cirúrgico são fatores para infecção do sítio cirúrgico ${ }^{(24-25)}$. Dentre estes fatores, a obesidade, pode ser passível de redução por meio de programas pré-operatórios de perda de peso antes da cirurgia eletiva ${ }^{(22)}$.

Nesse contexto, pode-se estabelecer uma abordagem pré-operatória diferenciada para os pacientes diabéticos, com estratégias de controle glicêmico, pois a normoglicemia parece ter efeito protetor nos pacientes com infecção profunda no espaço do órgão após a cirurgia cardíaca ${ }^{(26-27)}$. Além disso, a redução dos níveis de glicemia oportuniza a cicatrização mais rápida contribuindo para menor incidência de infecção(28).

No homem, pode haver maior tensão na ferida esternal, tornando o sexo masculino mais susceptível a mediastinite ${ }^{(25)}$. Doenças pulmonares como a doença pulmonar obstrutiva crônica (DPOC) associado a hábitos de vida como o tabagismo podem favorecer a ocorrência de problemas ventilatórios que provocam maior instabilidade esternal, dificulta a cicatrização expondo o paciente ao risco de mediastinite ${ }^{(28)}$. 
Os resultados de uma pesquisa ${ }^{(16)}$ mostram que $80 \%$ dos cateteres de sucção utilizados no intraoperatório de cirurgias de substituição da valva aórtica apresentavam contaminação bacteriana, sendo o agente etiológico mais frequente o Estafilococos coagulase negativa, o mesmo autor inferiu que o tempo de sucção não aumentou o risco de contaminação dos cateteres. A contaminação por esse tipo de bactéria também foi encontrada em outros estudos ${ }^{(29-30)}$ que investigaram infecção do sítio cirúrgico em transplantes cardíacos e revascularização do miocárdio, respectivamente.

Apesar da bactéria estafilococos coagulase negativa ser comumente encontrada na microbiota normal da pele, essa contaminação também pode derivar do pessoal na sala de operação ${ }^{(31)}$. Nessa perspectiva, os enfermeiros, considerados precursores na organização e sistematização do cuidado seguro ao paciente no centro cirúrgico, devem disseminar orientações de cautela entre todos os membros da equipe nos cuidados de assepsia das mãos, do sítio cirúrgico, na paramentação adequada, desinfecção de superfícies, equipamentos, utensílios e na manipulação dos mesmos durante o período transoperatório, evitando contaminações e contribuindo para redução de mediastinite e outras complicações no PO.

Os sinais para identificação precoce de mediastinite no PO, são conhecidos como o extravasamento de secreção purulenta no sítio cirúrgico, a instabilidade esternal e febre alta persistente, todavia parece haver poucas estratégias, além da preparação do paciente e da profilaxia antibiótica, que podem ser implementadas para reduzir a mediastinite ${ }^{(32)}$.

Outros aspectos relevantes a discutir são condições inerentes ao paciente, como a resistência antimicrobina e a incidência secundária de mediastinite, naqueles submetidos à cirurgias prévias e acometidos pela mediastinite. Um estudo mostra que o Estafilococcus aureus, foi observada em $60 \%$ dos episódios de mediastinite em pacientes submetidos à cirurgia cardíaca, porém a mortalidade, a duração da ventilação mecânica e o tempo de permanência na unidade de terapia intensiva foram significativamente maiores para pacientes com mediastinite secundária, ou seja, para aqueles que já haviam apresentado a infecção ${ }^{(19)}$.

Um estudo recente ${ }^{(21)}$ utilizou um escore de risco para prever mortalidade e complicações em pacientes submetidos a cirurgias cardíacas; os pacientes submetidos a trocas valvares e revascularização do miocárdio apresentaram um perfil de risco aumentado tanto para mortalidade hospitalar quanto para ocorrência de complicações como o acidente vascular encefálico e a mediastinite.

O tempo de internação pré-operatória também foi considerado um fator de risco independente para mediastinite em um estudo de coorte retrospectiva em pacientes cardíacos, levando a um aumento de risco de $15 \%$ de mediastinite por semana de permanência. $O$ atraso na realização do procedimento cirúrgico, muitas vezes em decorrência da alta demanda de pacientes e baixa disponibilidade de leitos de terapia intensiva no PO aliado a outros fatores (hipertensão pulmonar, diabetes, obesidade, distúrbios de filtração glomerular), foram considerados como riscos modificáveis para a mediastinite ${ }^{(20)}$.

Dois estudos ${ }^{(17-18)}$ comparam o desfecho PO de pacientes submetidos a MTD ou a EM para correção de valva mitral.

De acordo com os resultados a taxa de mediastinite foi menor em pacientes submetidos à MTD ${ }^{(17)}$. Um estudo $^{(18)}$ corrobora um resultado similar, onde nenhum paciente submetido à MTD desenvolveu mediastinite $e$ $2 \%$ dos pacientes submetidos à EM desenvolveram esta infecção. 
Recentemente, outro estudo mostrou que a ocorrência de mediastinite após troca valvar aórtica foi menor em pacientes sob técnica cirúrgica MTD quando comparados aos submetidos a EM, além disso, infere que pode haver diminuição da hemorragia pós-operatória e necessidade de transfusão de sangue, menor ocorrência de fibrilação atrial, menor índice de morbidade e menor tempo de internação hospitalar e unidade de terapia intensiva ${ }^{(33)}$.

Pressupõe-se que prover conhecimentos sobre elementos relacionados à ocorrência de mediastinite em pacientes submetidos à cirurgia de correção de válvulas, pode preparar a enfermagem para cuidados clínicos mais efetivos no perioperatório, desde a admissão hospitalar, explorando os antecedentes patológicos do paciente, munindo-o de informações preparatórias para o enfrentamento do PO e refletindo ações durante o processo cirúrgico com a equipe, a fim de minimizar o risco de complicações.

Diante deste cenário e considerando a mediastinite como uma complicação que implica altos índices e morbidade e mortalidade, custos ao sistema de saúde ${ }^{(34)}$, além de afligir a qualidade de vida do paciente ${ }^{(35)}$, fazse necessário atentar-se para as particularidades envolvendo as cirurgias valvares e sua ocorrência, a fim de motivar estudos futuros com vistas à prevenção e redução deste agravo no PO.

Em geral, a maioria das instituições hospitalares, juntamente com o enfermeiro assistencial, atuam no controle da infecção hospitalar devido à proximidade e acompanhamento da evolução clínica do paciente. $\mathrm{O}$ processo de enfermagem engloba além da observação do quadro clínico, a elaboração dos diagnósticos de enfermagem, estabelecimento de metas e implementação das ações necessárias ao cuidado do paciente, alimentação de dados à vigilância epidemiológica e à comissão de controle de infecção hospitalar, execução de treinamentos e educação continuada e busca contínua de resolutividade assistencial(36).

Espera-se que o conhecimento acerca desta lacuna seja mais explorado em estudos prospectivos, com o objetivo de fornecer subsídios para elaborar melhores estratégias durante os cuidados destinados aos pacientes submetidos à troca ou plastia das válvulas. Muitos destes cuidados podem ser viabilizados pela equipe de enfermagem que representa um elo entre o paciente e os demais integrantes da equipe multidisciplinar, além de acompanhar o paciente em todas as fases do perioperatório, os enfermeiros são capazes de identificar possíveis fatores que contribuem para a ocorrência deste agravo por meio da escuta às queixas do paciente, do exame físico, observação da ferida operatória e dos sinais e sintomas que denotam a evolução do paciente hospitalizado e os risco para complicações.

\section{CONCLUSÃO}

As evidências acerca da mediastinite em pacientes submetidos às cirurgias para correção de valvopatias, mostram que pode ocorrer contaminação bacteriana de cateteres utilizados durante o procedimento cirúrgico que favorecem o aparecimento desta complicação, ademais a escolha da técnica de abordagem cirúrgica, como a MTD, pode reduzir a ocorrência de mediastinite, bem como o tempo de permanência hospitalar. Espera-se que este estudo possa estimular a equipe de saúde à discussão a fim de melhorar a implementação das ações na prática clínica, prevenindo complicações, e auxiliar na construção de novos estudos acerca desta temática. 


\section{REFERÊNCIAS}

1. Datasus [Internet]. Brasília: Ministério da Saúde; 2017 [Acesso em 06 mar. 2017]. Disponível em: //www.datasus.gov.br.

2. Guaragna JC, Facchi LM, Baião CG, Cruz IB, Bodanese LC, Albuquerque L, et al. Predictors of mediastinitis after cardiac surgery. Rev Bras Cir Cardiovasc [Internet]. 2004 [Cited 2017 Mar 06]; 19(2):165-170. Available from: http://dx.doi.org/10.1590/S010276382004000200011

3. Campagnucci VP, Silva AMRP, Pereira WP, Chamlian EG, Gandra SMA, Rivetti LA. EuroSCORE and the patients undergoing coronary bypass surgery at Santa Casa de São Paulo. Rev Bras de Cirurgia Cardiovasc [Internet].; 2008 [Cited 2017 Mar 06]; 23(2):262-7. Available from: http://dx.doi.org/10.1590/S0102-76382008000200017

4. Abelha FJ, Botelho M, Fernandes V, Barros H. Outcome and quality of life after aorto-bifemoral bypass surgery. BMC Cardiovascular Disorders [Internet]. 2010 [Cited 2017 Mar 06]; 10:15. Available from: http://www.biomedcentral.com/1471$\underline{2261 / 10 / 15}$

5. Laizo A, Delgado FEdF, Rocha GM. Complications that increase the time of Hospitalization at ICU of patients submitted to cardiac surgery. Braz. J. Cardiovasc. Surg [Internet]. 2010 [Cited 2017 Mar 06]; 25(2):166-71. Available from:

http://dx.doi.org/10.1590/S0102-76382010000200007

6. Magedanz EH, Bodanese LC, Guaragna JCVdC, Albuquerque LC, Martins V, Minossi SD, et al. Risk score elaboration for mediastinitis after coronary artery bypass grafting. Brazilian Journal of Cardiovascular Surgery [Internet]. 2010 [Cited 2017 Mar 06]; 25(2):154-9. Available from: http://dx.doi.org/10.1590/S0102-76382010000200005

7. Garner JS, Jarvis WR, Emori TG, Horan TC, Hughes JM. CDC definitions for nosocomial infections, 1988. Am J Infect Control. 1988; 16(3):128-40.

8. Horan TC, Andrus M, Dudeck MA. CDC/NHSN surveillance definition of health care-associated infection and criteria for specific types of infections in the acute care setting. Am J Infect Control. 2008; 36(5):309-32.

9. lung B, Vahanian A. Epidemiology of valvular heart diasese in the adult. Nature Reviwes Cardiology. Nature Reviews Cardiology. 2011; 8:162-72.

10. Varadarajan P, Kapoor N, Bansal RC, Pai RG. Clinical profile and natural history of 453 nonsurgically managed patients with severe aortic stenosis. Ann Thorac Surg [Internet]. 2006 [Cited 2017 Mar 06]; 82(6):2111-5. Available from:

http://dx.doi.org/10.1016/j.athoracsur.2006.07.048

11. Mendes KDS, Silveira RCCP, Galvão CM. Integrative literature review: a research method to incorporate evidence in health care and nursing. Texto contexto - enferm [Internet]. 2008 [Cited 2017 Mar 06]; 17(4):758-64. Available from:

http://dx.doi.org/10.1590/S0104-07072008000400018

12. Melnyk BM, Fineout-Overholt E, Stillwell SB, Williamson KM.Evidence-based practice: step by step: the seven steps of evidencebased practice. Am J Nurs. 2010; 110(1):51-3.

13. Ursi ES, Gavão CM. Prevenção de lesões de pele no perioperatório: revisão integrativa da literatura. Rev. Latino-Am. Enfermagem [Internet]. 2006 [Cited 2017 Mar 06]; 14(1): 124-31. Available from: http://dx.doi.org/10.1590/S010411692006000100017.

14. Moher D, Shamseer L, Clarke M, Davina G, Liberati A, Petticrew M, et al. Preferred reporting items for systematic review and meta-analysis protocols (PRISMA-P) 2015 statement. Syst Rev [Internet]. 2015 [Cited 2017 Mar 06]; 4:1. Available from:

http://www.systematicreviewsjournal.com/content/4/1/1

15. Thourani VH, Ailawadi G, Szeto WY, Dewey TM, Guyton RA, Mack MJ, et al. Outcomes of surgical aortic valve replacement in high-risk patients: a multiinstitutional study. Ann Thorac Surg [Internet]. 2011 [Cited 2017 Mar 06]; 91(1):49-56. Available from: http://dx.doi.org/10.1016/j.athoracsur.2010.09.040

16. Larsson J, Sutherland S, Söderström A, Emanuel CR, Jeppsson A, Olofsson EH, et al. Bacterial contamination of suction catheter tips during aortic valve replacement surgery: a prospective observational cohort study. Patient Saf Surg [Internet]. 2015 [Cited 2017 Mar 06]; 9:17. Available from: https://pssjournal.biomedcentral.com/articles/10.1186/s13037-015-0066-5

17. Nishi H, Miyata H, Motomura N, Toda K, Miyagawa S, Sawa Y, et al. Propensity-matched analysis of minimally invasive mitral valve repair using a nationwide surgical database. Surg Today. 2015; 45(9):1144-52.

18. Sansone F, Punta G, Parisi F, Dato GM, Zingarelli E, Flocco R, et al. Right minithoracotomy versus full sternotomy for the aortic valve replacement: preliminary results. Heart Lung Circ. 2012; 21(3):169-73.

19. Combes A, Trouillet JL, Baudot J, Mokhtari M, Chastre J, Gibert C. Is it possible to cure mediastinitis in patients with major postcardiac surgery complications? Ann Thorac Surg [Internet]. 2001 [Cited 2017 Mar 06]; 72(5):1592-7. Available from:

http://dx.doi.org/10.1016/S0003-4975(01)03087-9

20. Sang SLW, Chaturvedi R, Alam A, Samoukovic G, Varennes B, Lachapelle K. Preoperative hospital length of stay as a modifiable risk factor for mediastinitis after cardiac surgery. Journal of Cardiothoracic Surgery [Internet]. 2013 [Cited 2017 Mar 06]; 8:45. Available from: https://cardiothoracicsurgery.biomedcentral.com/articles/10.1186/1749-8090-8-45

21. Ensminger S, Fujita B, Landwehr S, Bauer T, Möllmann H, Beckmann A, et al. Current Trends in Practice and Outcomes after Surgical Aortic Valve Replacement in Germany: Update of the GARY Registry. Thorac cardiovasc Surg. 2017; 65(S01):S1-S110.

22. Rehman SM, Elzain O, Mitchell J, Shine B, Bowler IC, Sayeed R, et al. Risk factors for mediastinitis following cardiac surgery: the importance of managing obesity. J Hosp Infect. 2014; 88(2):96-102. 
23. Tiveron MG, Fiorelli Al, Mota EM, Mejia OAV, Brandão CMdA, Dallan LA, et al. Preoperative risk factors for mediastinitis after cardiac surgery: analysis of 2768 patients. Braz. J. Cardiovasc. Surg [Internet]. 2012 [Cited 2017 Mar 06]; 27(2):203-10. Available from: http://dx.doi.org/10.5935/1678-9741.20120035

24. Olsen PS, Faraz M, Jakobsen S, Steinbrüchel DA. Cardiac surgery in octogenarians. Ugeskr Laeger. 2003; 165(24):2487-90. 25. Abboud CS, Wey SB, Baltar VT. Risk factors for mediastinitis after cardiac surgery. Ann Thorac Surg [Internet]. 2004 [Cited 2017 Mar 06]; 77(2):676-83. Available from: http://dx.doi.org/10.1016/50003-4975(03)01523-6

26. Mathew I, Grimes MC, Rao RH, Cunningham CA, Muder R, Perreiah PL. Eliminating Deaths from Mediastinitis after Cardiac Surgery Using Aggressive Glycemic Intervention to Maintain Blood Glucose 80-140mg/DI: The VA Pittsburgh Experience: Diabetes Clinical Care; Genetics \& Epidemiology. Endocrine Society's 96 ${ }^{\text {th }}$ Annual Meeting Chicago, 014.

27. Bower WF, Cheung CS, Lai RW, Underwood MJ, van Hasselt CA. An audit of risk factors for wound infection in patients undergoing coronary artery bypass grafting or valve replacement. Hong Kong Med J [Internet]. 2008 [Cited 2017 Mar 06]; 14(5):371-8. Available from: http://www.hkmj.org/abstracts/v14n5/371.htm

28. Loop FD, Lytle BW, Cosgrove DM, Mahfood S, McHenry MC, Goormastic M, et al. Sternal wound complications after isolated coronary artery bypass grafting: early and late mortality, morbidity, and cost of care. Ann Thorac Surg [Internet]. 1990 [Cited 2017 Mar 06]; 49(2):179-86. discussion 179-87. Available from: http://dx.doi.org/10.1016/0003-4975(90)90136-T

29. Rodrigues JASDN, Rebustini RELF, Poveda VB. Surgical site infection in patients submitted to heart transplantation. Rev. LatinoAm. Enfermagem [Internet]. 2016 [Cited 2017 Mar 06]; 24(e2700). Available from: http://dx.doi.org/10.1590/1518-8345.0821.2700 30. Egito JGTd, Abboud CS, Oliveira APVd, Máximo CAG, Montenegro CM, Amato VL, et al. Clinical evolution of mediastinitis in patients undergoing adjuvant hyperbaric oxygen therapy after coronary artery bypass surgery. Einstein [Internet]. 2013 [Cited 2017 Mar 06]; 11(3):345-9. Available from: http://www.scielo.br/scielo.php?pid=S1679-

$45082013000300014 \&$ script=sci abstract\&tlng=pt

31. Owens CD, Stoessel K. Surgical site infections: epidemiology, microbiology and prevention. J Hosp Infect. 2008; 70(2):3-10.

32. Gelape CL. Surgical wound infection following heart surgery. Arq Bras Cardiol. [Internet]. 2007 [Cited 2017 Mar 06]; 89(1):e3-e9. Available from: http://dx.doi.org/10.1590/S0066-782X2007001300013

33. Stoliński J, Plicner D, Grudzién G, Wąsowicz M, Musial R, Andres J, et al. A comparison of minimally invasive and standard aortic valve replacement. The Journal of Thoracic and Cardiovascular Surgery. 2016; 152(4):1030-1039.

34. Greco G, Shi W, Michler RE, Meltzer DO, Ailawadi G, Hohmann SF, et al. Costs associated with health care-associated infections in cardiac surgery. J Am Coll Cardiol [Internet]. 2015 [Cited 2017 Mar 06]; 65(1):15-23. Available from:

http://www.sciencedirect.com/science/article/pii/S0735109714068466?via\%3Dihub

35. Arnold SV, Spertus JA, Vemulapalli S, Li Z, Matsouaka RA, Baron SJ, et al. Quality-of-Life Outcomes After Transcatheter Aortic Valve Replacement in an Unselected Population: A Report From the STS/ACC Transcatheter Valve Therapy Registry. JAMA Cardiology. 2017; 2(4):409-16.

36. Magalhães MGPA, Alves LMO, Alcantara LFM, Bezerra SMMS. Post-operative mediastinitis in a heart hospital of Recife: contributions for nursing care. Rev esc enferm USP [Internet]. 2012 [Cited 2017 Mar 06], 46(4):865-71. Available from: http://dx.doi.org/10.1590/S0080-62342012000400012. 